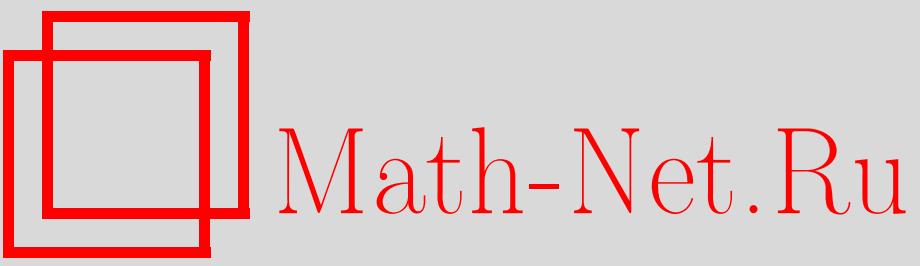

В. В. Козлов, Весовые средние, равномерное распределение и строгая эргодичность, УМН, 2005, том 60, выпуск 6, 115-138

DOI: https://doi.org/10.4213/rm1679

Использование Общероссийского математического портала Math-Net.Ru подразумевает, что вы прочитали и согласны с пользовательским соглашением

http://www . mathnet.ru/rus/agreement

Параметры загрузки:

IP : 54.81 .137 .203

26 апреля 2023 г., 06:43:20 


\title{
ВЕСОВЫЕ СРЕДНИЕ, РАВНОМЕРНОЕ РАСПРЕДЕЛЕНИЕ И СТРОГАЯ ЭРГОДИЧНОСТЬ
}

В. В. Козлов

\begin{abstract}
Рассматривается круг вопросов, связанный с применением методов суммирования Рисса и Вороного в эргодической теории, теории чисел и теории вероятностей. Обсуждается парадокс первых цифрр, указаны усиления классического результата $\Gamma$. Вейля о равномерном распределении дробных долей значений многочлена, рассматривается возможность усиления эргодической теоремы Биркгофа-Хинчина. В заключение перечислены некоторые нерешенные задачи.

Библиография: 44 названия.
\end{abstract}

\section{СОДЕРЖАНИЕ}

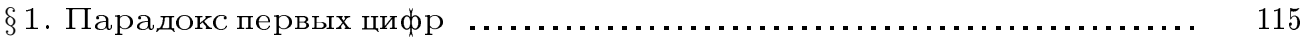

$\S 2$. Весовые средние и типы равномерного распределения ............... 119

$\S 3$. Равномерное распределение и сходимость по Вороному $\ldots . . . \ldots \ldots . .121$

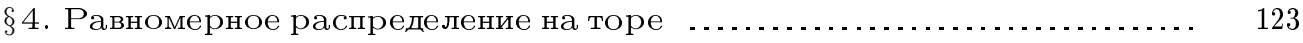

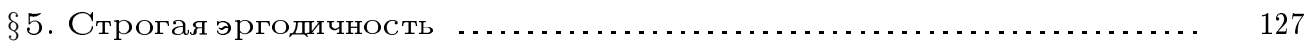

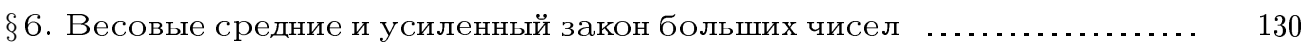

$\S 7$. Индивидуальная эргодическая теорема ......................... 133

$\S 8$. Некоторые нерешенные задачи $\ldots . \ldots \ldots \ldots \ldots \ldots \ldots \ldots \ldots \ldots \ldots \ldots \ldots . . \ldots \ldots$

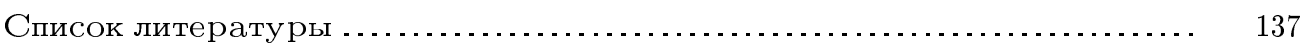

\section{1. Парадокс первых цифр}

Начнем с обсуждения известной задачи о частоте появления цифры $g(1 \leqslant g \leqslant 9)$ в последовательности первых цифр степеней двойки:

$2,4,8,1,3,6,1,2,5,1,2,4,8,1,3,6,1,2,5,1,2,4,8$,

$1,3,6,1,2,5,1,2,4,8,1,3,6,1,2,5,1, \ldots$.

Введем последовательность чисел $x_{n}(g)$ (или, кратко, $\left.x_{n}\right)$, которые равны 1 , если десятичное разложение $2^{n}$ начинается с цифры $g$, и равны нулю в противном случае. Частота появления $g$ определяется как

$$
\lim _{n \rightarrow \infty} \frac{\nu_{g}(n)}{n}
$$


где $\nu_{g}(n)=\sum_{1}^{n} x_{k}$. Хорошо известно, что этот предел сушествует и равен

$$
\lg \frac{1+g}{g} .
$$

В частности, в последовательности (1.1) семерка чаще встречается, чем восьмерка.

Этот результат можно сформулировать по-другому:

$$
x_{n} \rightarrow \lg \frac{1+g}{g}(C),
$$

где $C$ - сходимость по Чезаро.

Напомним вьвод формулы (1.2). Ниже мы используем его идею в другой ситуации. Ясно, что десятичная запись числа $2^{n}$ начинается с цифры $g$, если

$$
g \cdot 10^{k} \leqslant 2^{n}<(g+1) \cdot 10^{k}
$$

при некотором целом $k \geqslant 0$. Логарифмируя эти неравенства и переходя к дробньм частям, получаем неравенства

$$
\lg g \leqslant\{n \lg 2\}<\lg (g+1) .
$$

Так как число $\lg 2$ иррационально, то последовательность дробных долей $\{n \lg 2\}$ равномерно распределена на отрезке [0, 1] в смысле классического определения Вейля. Но это и означает, что в среднем числа $\{n \lg 2\}$ попадают в интервал $[\lg g, \lg (g+1))$ с частотой, равной длине этого интервала.

Упростим теперь задачу, заменив $2^{n}$ на $n$. Спрашивается, с какой частотой числа натурального ряда начинаются с цифры $g$ ? В этом случае неравенство (1.3) надо заменить неравенством

$$
\lg g \leqslant\{\lg n\}<\lg (g+1) .
$$

Известно, что хотя последовательность дробных долей логарифмов всюду плотна в единичном отрезке, она не является равномерно распределенной. Этот результат установил О. Ж. Френель еше в 1917 г. Следовательно, в данном случае отношение $\nu_{g}(n) / n$ (средняя частота появления цифры $g$ ) вообще не имеет предела, когда $n \rightarrow \infty$.

Снова введем последовательность чисел $x_{n}$, которые равны 1 , если десятичное разложение $n$ начинается с цифры $g$, и равны 0 в противном случае.

TEOPEMA 1.

$$
x_{n} \rightarrow \lg \frac{1+g}{g} \quad(R, 1 / n) .
$$

Здесь $(R, 1 / n)$ обозначает логарифмический метод суммирования: $s_{n} \rightarrow s(R, 1 / n)$, если

$$
\lim _{n \rightarrow \infty} \frac{s_{1}+s_{2} / 2+\cdots+s_{n} / n}{1+1 / 2+\cdots+1 / n}=\lim _{n \rightarrow \infty} \frac{1}{\ln n} \sum_{k=1}^{n} \frac{s_{k}}{k}=s .
$$

Этот метод включает метод Чезаро: если $s_{n} \rightarrow s(C)$, то $s_{n} \rightarrow s(R, 1 / n)$.

Теорема 1 доказана Р.Л. Дунканом [1]. Этой теореме предшествовал более ранний результат Б. Флейнджер [2], которая вместо риссовских средних использовала итерации метода Чезаро. Следует отметить, что теорема 1 - весьма частный случай результатов более ранней работы [3] о свойствах равномерного распределения, когда метод Чезаро заменяется методами суммирования Рисса (об этом речь пойдет в $§ 2$ ). 
Теорема 1 служит обоснованием парадокса первой цифры: вопреки ожиданию наугад взятое натуральное число почти в семь раз чаще начинается с единицы, чем с девятки. Этот парадокс обычно связывают с именем $\Phi$. Бенфорда, американского физика, заметившего однажды, что в книге, содержашей подробную таблицу логариффмов, наиболее загрязнены края страниц в ее начале [4]. Это означает, что люди чаще всего ищут логарифмы чисел, которые начинаются с 1 , и реже всего - логарифмы чисел, начинающиеся с 9. В.И. Арнольд также заметил, что распределение первых цифр в таблицах населения и площадей стран мира соответствует закону Бенфорда (см. комментарии в книге [5]).

Следует отметить, что закон Бенфорда был открыт и опубликован американским астрономом Саймоном Ньюкомом шестьюдесятью годами раньше [6]. Ньюком приводит следующую таблицу частот появления различных цифр на первом и втором месте в десятичной записи натуральных чисел.

$\begin{array}{cccc}\text { цифра } & & \begin{array}{c}\text { первая } \\ \text { цифра }\end{array} & \begin{array}{c}\text { вторая } \\ \text { цифра }\end{array} \\ 0 & \ldots & \ldots & 0.1197 \\ 1 & \ldots & 0.3010 & 0.1139 \\ 2 & \ldots & 0.1761 & 0.1088 \\ 3 & \ldots & 0.1249 & 0.1043 \\ 4 & \ldots & 0.0969 & 0.1003 \\ 5 & \ldots & 0.0792 & 0.0967 \\ 6 & \ldots & 0.0669 & 0.0934 \\ 7 & \ldots & 0.0580 & 0.0904 \\ 8 & \ldots & 0.0512 & 0.0876 \\ 9 & \ldots & 0.0458 & 0.0850\end{array}$

Эти частоты Ньюком вычисляет, исходя из предположения, что последовательность $\{\lg n\}$ равномерно распределена на отрезке [0,1]. Как мы видели, само определение равномерно распределенной последовательности нуждается в уточнении. Ньюком не дает точных определений. Но при этом надо помнить, что его пионерская работа опубликована за 35 лет до классической работы Германа Вейля о равномерном распределении по модулю 1.

Из таблищы Ньюкома видно, что вторые цифры в десятичной записи натуральных чисел также распределены неравномерно: ноль встречается в 1.4 раза чаще, чем девятка. Частота появления цифры $g(0 \leqslant g \leqslant 9)$ на втором месте подсчитьвается тем же способом. Десятичные разложения таких чисел имеют вид

$$
1 g \ldots, 2 g \ldots, \ldots, 9 g \ldots
$$

Надо подсчитать частоты появления этих чисел (например, в смысле логарифмической сходимости) и затем эти частоты сложить.

Частота, с которой встречаются числа с десятичной записью $\mathrm{kg} . .$, равна

$$
\lg \frac{10 k+g+1}{10 k+g} .
$$

Следовательно, частоты появления цифр $0,1, \ldots, 9$ на втором месте равны

$$
\lg \frac{11}{10} \cdot \frac{21}{20} \cdots \frac{91}{90}, \ldots, \lg \frac{20}{19} \cdot \frac{30}{29} \cdots \frac{100}{99} .
$$


Аналогично решается задача о вычислении частот появления цифр $0,1, \ldots, 9$ на третьем месте:

$$
\lg \frac{101}{100} \cdot \frac{111}{110} \cdot \frac{121}{120} \ldots \frac{991}{990}, \ldots, \lg \frac{110}{109} \cdot \frac{120}{119} \cdot \frac{130}{129} \ldots \frac{1000}{999} .
$$

Эти числа отличаются друг от друга еще более незначительно.

ТЕОРема 2. Частота появления каждой иифры на $n$-м месте стремится к $1 / 10$ при $n \rightarrow \infty$.

Родственное по своему смыслу утверждение отмечено ранее в [7]. Пусть $D(n)-$ количество всех цифр всех натуральных чисел, не превосходяших $n$, а $D_{g}(n)$ - количество появлений цифры $g$ в десятичной записи всех таких чисел. Как показано в [7],

$$
D_{g}(n) / D(n) \rightarrow 1 / 10
$$

Теорема 2 формально не зависит от этого результата, поскольку частота появления цифры $g$ определяется в (1.4) с помощью сходимости по Чезаро, в то время как теорема 2 оперирует с частотами, определение которых использует более сильные методы суммирования.

Соотношение (1.4) фактически было известно до работы [7]. Например, для двоичных разложений оно содержится в учебнике [8] в качестве упражнения в приложении к гл. V.

Докажем теорему 2. Для определенности рассмотрим частоту появления нуля:

$$
\lg \left(1+\frac{1}{10^{n}}\right)\left(1+\frac{1}{10^{n}+10}\right) \cdots\left(1+\frac{1}{10^{n+1}-10}\right) .
$$

Добавим (для удобства записи) к (1.5) слагаемое

$$
\lg \left(1+\frac{1}{10^{n+1}}\right)
$$

которое экспоненциально быстро стремится к нулю при $n \rightarrow \infty\left(\right.$ как $\left.10^{-n}\right)$. В итоге сумма (1.5) и (1.6) имеет вид

$$
s_{n}=\sum_{j=0}^{9 \cdot 10^{n-1}} \lg \left(1+\frac{1}{10^{n}+10 j}\right)
$$

Воспользуемся элементарным неравенством: если $x>0$, то

$$
0<x-\ln (1+x)<x^{2} / 2 .
$$

Следовательно,

$$
0<u_{n}-s_{n}<v_{n},
$$

где

$$
u_{n}=\frac{1}{10 \ln 10} \sum_{j=0}^{9 \cdot 10^{n-1}} \frac{1}{10^{n-1}+j}, \quad v_{n}=\frac{1}{2 \ln 10} \sum_{j=0}^{9 \cdot 10^{n-1}} \frac{1}{\left(10^{n}+10 j\right)^{2}}
$$


Далее,

$$
\int_{0}^{9 \cdot 10^{n-1}} \frac{d x}{10^{n-1}+x}<\sum_{j=0}^{9 \cdot 10^{n-1}} \frac{1}{10^{n-1}+j}<\int_{0}^{9 \cdot 10^{n-1}} \frac{d x}{x-1+10^{n-1}} .
$$

Интегралы слева и справа равны соответственно

$$
\ln 10 \text { и } \lg \frac{10^{n}-1}{10^{n-1}-1}=\ln \left(10+\frac{9}{10^{n-1}-1}\right) .
$$

Следовательно, $u_{n} \rightarrow 1 / 10$ при $n \rightarrow \infty$.

Так как

$$
\sum_{j=0}^{9 \cdot 10^{n-1}} \frac{1}{\left(10^{n}+10 j\right)^{2}}<\frac{9 \cdot 10^{n-1}+1}{10^{2 n}}<\frac{1}{10^{n}},
$$

то $v_{n} \rightarrow 0$ при $n \rightarrow \infty$. Но тогда из (1.7) вытекает, что $s_{n} \rightarrow 1 / 10$. Что и требовалось.

Как видно из доказательства, разность между (1.5) и $1 / 10$ убьвает с ростом $n$ как $10^{-n}$. Таким образом, при больших $n$ парадокс $n$-ой цифры практически не заметен.

Обсуждение различных аспектов парадокса первых цифрр можно найти в работах $[7],[9]$.

\section{§ 2. Весовые средние и типы равномерного распределения}

Соображения предыдущего параграфа, связанные с заменой сходимости средних арифметических (по Чезаро) другими методами суммирования, приводят нас к естественному обобщению равномерно распределенных последовательностей.

Пусть $x_{n}(n \geqslant 1)$ - последовательность точек единичного отрезка, $L \subset[0,1]$ - произвольньй отрезок длины $l, f$ - характеристическая функция этого отрезка. Пусть $S$ - некоторьй линейный и регулярный метод суммирования.

Будем говорить, что последовательность точек $x_{n} S$-равномерно распределена по модулю 1 (или, более кратко, $S$-рр), если для каждого отрезка $L$

$$
f\left(x_{n}\right) \rightarrow \operatorname{mes} L=l \quad(S) .
$$

Если $S$ - метод Чезаро, то получаем классическое определение Вейля.

Легко доказывается, что соотношение (2.1) справедливо для всех интегрируемых по Риману функций $f$, только надо, конечно, $l$ заменить интегралом от $f$ по отрезку $0 \leqslant x \leqslant 1$. Как и в классическом случае, справедливо обращение этого результата: если для всех целых $m \neq 0$

$$
e^{2 \pi i m x_{n}} \rightarrow 0 \quad(S),
$$

то последовательность $x_{n}$ будет $S$-рр. Ясно, что всякая $S$-рр последовательность всюду плотно заполняет единичньй отрезок.

Последовательности, равномерно распределенные в обобщенном смысле, на самом деле уже рассматривал сам Г. Вейль в классической работе "О равномерном распределении чисел по модулю один” (см. [5]). В качестве метода суммирования $S$ он рассматривает методы Рисса $\left(R, p_{n}\right)$, у которых весовые коэффициенты $p_{n}$ монотонно убьвают и $\sum p_{n}=\infty$, либо монотонно возрастают и при этом

$$
(n+1) p_{n}=O\left(\sum_{1}^{n} p_{j}\right) .
$$


Вейль доказывает (см. [5; теорема 10, с. 74]) следующее. Пусть $P(x)$-многочлен, у которого хотя бы один коэффициент (кроме свободного) иррационален. Тогда при сделанных выше предположениях относительно весовых коэффициентов $p_{n}$ и для любой интегрируемой по Риману функции $f$

$$
f(P(n)) \rightarrow \int_{0}^{1} f(x) d x \quad\left(R, p_{n}\right) .
$$

Это утверждение для линейной формы $P(x)$ и монотонно убьвающей последовательности $p_{n}$ содержится также в книге Г. Полиа и Г. Сеге [10; задача 173].

Однако эти утверждения не содержат ничего нового по сравнению с результатом о равномерном распределении по модулю 1 последовательности $\{P(n)\}$ (это - теорема 9 из [5], с. 69). Дело в том, что при $p_{n+1} \leqslant p_{n}$ справедливо следуюшее свойство: если $s_{n} \rightarrow s(C)$, то $s_{n} \rightarrow s\left(R, p_{n}\right)$. Это вытекает из теоремы Чезаро [11; теорема 14], установленной в 1888 г. (за 25 лет до работы Вейля). Другими словами, при сделанных предположениях метод Рисса включает метод Чезаро. С другой стороны, если $p_{n+1} \geqslant p_{n}$, то, наоборот, метод Чезаро всегда включает метод Рисса [11; теорема 14]. Если, кроме того, вьполнено условие (2.2), то эти методы равносильны. Таким образом, предельное соотношение (2.3) будет содержательным только для тех методов Рисса, у которых $p_{n}$ возрастают, но свойство (2.2) не вьполняется.

Отметим, что при вьполнении (2.2) $p_{n}$ могут возрастать как степени $n$. С другой стороны, если $p_{n}$ возрастают экспоненциально быстро, то метод Рисса теряет свою силу и становится эквивалентным обычной сходимости [11; теорема 15]. Поэтому реальный интерес в этом обобщении представляют случаи, когда, например, $p_{n} \sim \exp n^{\gamma}, 0<\gamma<1$.

Укажем достаточные условия $R$-рр.

ТеОРема 3. Пусть непрерывно дифферениируемые функиии $f(x), g(x)(x \geqslant 1)$ удовлетворяют следующим условиям:

1) $f^{\prime} \neq 0 u f^{\prime}(x) \rightarrow 0$ nрu $x \rightarrow \infty$

2) функиия $g>0$ либо не убьвает $и$

$$
\frac{g(x)}{\int_{1}^{x} g(t) d t} \rightarrow 0 \quad \text { npu } \quad x \rightarrow \infty
$$

либо не возрастает $и$

$$
\int_{1}^{\infty} g(x) d x=\infty,
$$

3) отношение $g / f^{\prime}$ либо не возрастает, либо не убъвает и

$$
\frac{g(x)}{f^{\prime}(x) \int_{1}^{x} g(t) d t} \rightarrow 0 \quad \text { npu } \quad x \rightarrow \infty .
$$

Тогда последовательность $\{f(n)\}$ будет $(R, g(n))$-равномерно распределенной.

Эта теорема при несколько иных предположениях $\left(f(x) \nearrow \infty\right.$ и $\left.f^{\prime}(x) \searrow 0\right)$ доказана в [3]. Приведенный вариант утверждения о $R$-рр указан в [12]. Доказательство теоремы 3 использует формулу суммирования Эйлера-Маклорена.

Из этой теоремы вьводится ряд полезных следствий. 
СлЕДСТВИЕ 1. В предположсениях теоремы 3 последовательность $\{f(n)\}$ всюду плотна на единичном отрезке.

СлЕДСТВИЕ 2 (теорема Фейера [10], [13]). Если $f^{\prime}(x)$ монотонно стремится $\kappa$ нулю и $x\left|f^{\prime}(x)\right| \rightarrow \infty$ при $x \rightarrow \infty$, то последовательность $\{f(n)\}$ равномерно распределена по Вейлю.

СлеДСТВИЕ 3. Пусть $f^{\prime}>0, f^{\prime}(x) \searrow 0 u f(x) \rightarrow \infty n p u x \rightarrow \infty$. Тогда $\{f(n)\}-$ $\left(R, f^{\prime}(n)\right)$-равномерно распределенная последовательность.

Надо положить $g=f^{\prime}$ и воспользоваться теоремой 3 . В частности, последовательность $\{c \ln n\}, c \neq 0$, будет $(R, 1 / n)$-рр.

\section{§ 3. Равномерное распределение и сходимость по Вороному}

Кроме сходимости по Риссу мы будем использовать сходимость по Вороному. Пусть снова $p_{1}>0, p_{n} \geqslant 0$. Для последовательности $s_{n}(n \geqslant 1)$ положим

$$
u_{n}=\frac{p_{n} s_{1}+p_{n-1} s_{2}+\cdots+p_{1} s_{n}}{p_{1}+p_{2}+\cdots+p_{n}} .
$$

Ясно, что если $s_{i}=s_{1}$, то $u_{n}=s_{1}$. Если $u_{n} \rightarrow s$, то полагают $s_{n} \rightarrow s\left(W, p_{n}\right)$. Теория суммирования Вороного подробно изложена в [11].

В западной математической литературе метод Вороного называют методом Нёрлунда, которьй рассмотрел его спустя 18 лет после публикации заметки Г. Ф. Вороного 1901 года.

Критерий регулярности $W$-метода:

$$
\frac{p_{n}}{p_{1}+\cdots+p_{n}} \rightarrow 0
$$

Оказывается, всякие два регулярных метода Вороного совместимы: если $s_{n} \rightarrow s(W)$ и $s_{n} \rightarrow s^{\prime}\left(W^{\prime}\right)$, то $s=s^{\prime}$. Подчеркнем, что методы Рисса не обладают этим важным свойством.

Если $W$-метод регулярен и последовательность весовых коэффициентов $p_{n}$ не убывает, то метод $\left(W, p_{n}\right)$ включает метод Чезаро $C$. Больший интерес представляют условия обратного включения. Пусть $p_{1}=1, p_{n}>0$. Если $W$-метод регулярен, $p_{n}$ не возрастают и

$$
\frac{p_{n+1}}{p_{n}} \geqslant \frac{p_{n}}{p_{n-1}}
$$

для всех $n>2$, то $C$ включает $\left(W, p_{n}\right)$.

Имеется аналог теоремы 3 для равномерного распределения в смысле средних Вороного, отмеченньй в [12].

ТеОРема 4. Пусть непрерывно дифференцируемье функиии $f(x), g(x)(x \geqslant 1)$ удовлетворяют следующим условиям:

1) $f^{\prime} \neq 0 u f^{\prime}(x) \rightarrow 0$ при $x \rightarrow \infty$,

2) положительная функиия $g$ монотонна $u$

$$
\begin{gathered}
\int_{1}^{\infty} g(x) d x=\infty \\
\frac{g(x)}{\int_{1}^{x} g(t) d t} \rightarrow 0 \quad \text { npu } \quad x \rightarrow \infty
\end{gathered}
$$


3) отношение $g(n-x) / f^{\prime}(x)$ не убьвает или не возрастает на отрезке $1 \leqslant$ $x \leqslant n$,

4) $\left|f^{\prime}(x)\right| \int_{1}^{x} g(t) d t \rightarrow \infty$ nрu $x \rightarrow \infty$.

Тогда последовательность $\{f(n)\}$ будет $(W, g(n))$-рр на единичном отрезке.

Условие (3.1) - условие регулярности метода Вороного. Если положить $g(x)=1$, то получим известную теорему Фейера о равномерном распределении по Вейлю.

СледСтВИЕ. Пусть $f^{\prime}>0, f^{\prime}(x) \searrow 0, g(x)>0$ не возрастает $u$

$$
f^{\prime}(x) \int_{1}^{x} g(t) d t \rightarrow \infty
$$

при $x \rightarrow \infty$. Тогда $\{f(n)\}-(W, g(n))-p p$.

Положим, например, $g(x)=1 / x$. Тогда (3.2) принимает вид

$$
f^{\prime}(x) \ln x \rightarrow \infty \text {. }
$$

В этом случае последовательность $\{f(n)\}-(W, 1 / n)$-рp.

Особый интерес представляет случай, когда функция $g$ возрастает. Тогда регулярный $(W, g(n))$-метод включает метод Чезаро. Однако здесь (как правило) условие 3 ) теоремы 4 не выполняется. Укажем одну из возможных модификаций теоремы 4.

Будем считать далее, что функция $f$ дважды непрерьвно дифференцируема. Положим $\varphi(x)=1 / f^{\prime}(x)$. Если продифференцировать произведение $\varphi(x) g(n-x)$ по $x$ и разделить результат на это произведение, то получим

$$
\frac{\varphi^{\prime}(x)}{\varphi(x)}-\frac{g^{\prime}(n-x)}{g(n-x)} .
$$

Предположим, что функции $\varphi^{\prime} / \varphi$ и $g^{\prime} / g$ монотонно убывают до нуля при $x \rightarrow \infty$. В частности, $f^{\prime \prime} / f^{\prime} \rightarrow 0$ монотонно. Тогда при достаточно больших $n$ в интервале $[1, n]$ функция (3.3) имеет ровно один нуль, которьй мы обозначим $x_{n}$.

ТеОРема 5 [14]. Пусть выполнены следующие условия:

1) $f^{\prime}>0, f^{\prime}(x) \rightarrow 0, f^{\prime \prime}(x) / f^{\prime}(x)$ монотонно стремится $\kappa$ нулю при $x \rightarrow \infty$,

2) $g>0, g$ монотонно возрастает, $g^{\prime}(x) / g(x) \rightarrow 0$ монотонно при $x \rightarrow \infty$, 3)

$$
\frac{g(n)}{f^{\prime}\left(x_{n}\right) \int_{1}^{n} g(t) d t} \rightarrow 0, \quad \frac{g\left(x_{n}\right)}{f^{\prime}(n) \int_{1}^{n} g(t) d t} \rightarrow 0
$$

nрu $n \rightarrow \infty$.

Тогда последовательность $\{f(n)\}$ будет $(W, g(n))-p p$.

СлЕДСТВИЕ. Пусть $f^{\prime}>0$, функции $f^{\prime}(x)$ u $\frac{f^{\prime \prime}(x)}{f^{\prime}(x)}$ монотонно стремятся к нулю при $x \rightarrow \infty u$

$$
\frac{f^{\prime \prime}(x)}{f^{\prime 2}(x)} \rightarrow 0
$$

Тогда $\{f(n)\}$ будет $\left(W, 1 / f^{\prime}(n)\right)-p p$.

Надо положить $g(x)=1 / f^{\prime}(x)$. Легко проверить, что в этом случае все условия теоремы 5 вьполнены, причем $x_{n}=(n-1) / 2$. При этом условия (3.4) вытекают из (3.5). 
ПримеР. Положим $f(x)=\ln ^{\alpha} x, \alpha>0$. Условие (3.5) вьполняется лишш при $\alpha>1$. В этом случае последовательность $\left\{\ln ^{\alpha} n\right\}$ будет $W$-рр при подходяшем выборе регулярного $W$-метода (в качестве $W$ можно взять, например, метод Чезаро). Однако, это свойство теряется при $\alpha=1$.

ТеОрема 6 [14]. Пусть $a \geqslant 2$ - иелое $и W$-регулярный метод суммирования Вороного. Тогда последовательность

$$
\left\{\frac{1}{2} \log _{a} n\right\}
$$

не является $W-p p$.

Используя метод работы [14], Г.А. Калябин совсем недавно доказал, что этот результат справедлив для любой последовательности $\{\alpha \ln n\}, \alpha \neq 0$.

\section{$\S 4$. Равномерное распределение на торе}

Пусть $\mathbb{T}^{n}-n$-мерный тор с угловыми координатами $x=\left(x_{1}, \ldots, x_{n}\right)$, изменяющимися по $\bmod 2 \pi$. Движение на $\mathbb{T}^{n}-$ это непрерьвное отображение $t \mapsto x(t)$. Оно называется равномерно распределенным на $\mathbb{T}^{n}$ (по Вейлю), если для любой измеримой по Жордану области $D \subset \mathbb{T}^{n}$

$$
\lim _{T \rightarrow \infty} \frac{\nu_{D}(T)}{T}=\frac{\operatorname{mes} D}{\operatorname{mes} \mathbb{T}^{n}},
$$

где $\nu_{D}(T)$ - сумма длин интервалов на отрезке $[0, T]$, когда $x(t) \in D ;$ mes $\mathbb{T}^{n}=(2 \pi)^{n}$.

Вейль дал следующий критерий равномерного распределения:

$$
\lim _{T \rightarrow \infty} \frac{1}{T} \int_{0}^{T} e^{i(m, x(t))} d t=0
$$

для всех целочисленных векторов $m \neq 0$. Он же отметил следующий результат (теорема 8 из [5], с. 69): если $x_{1}(t), \ldots, x_{n}(t)$ - такие $n$ многочленов от $t$, что

$$
\sum m_{j} x_{j}(t) \neq \mathrm{const}
$$

для всех целых $m_{j}$, не равных одновременно нулю, то движение $x_{j}=x_{j}(t)(1 \leqslant j \leqslant n)$ равномерно распределено на $\mathbb{T}^{n}$.

Пусть $t \mapsto \lambda(t)$ - положительная непрерьвная функция, причем

$$
\int_{0}^{\infty} \lambda(t) d t=\infty
$$

Будем говорить, что $f(t) \rightarrow \bar{f}(R, \lambda)$, если

$$
\int_{0}^{T} \lambda(t) f(t) d t / \int_{0}^{T} \lambda(t) d t \rightarrow \bar{f}
$$

при $T \rightarrow \infty$. Это - непрерьвньй аналог метода суммирования Рисса. Укажем основные свойства $(R, \lambda)$-метода, не стремясь к законченности и полноте.

Во-первых, $(R, \lambda)$-метод линеен и регулярен. Последнее свойство - прямое следствие правила Лопиталя. 
Далее, пусть имеется еще одна функция $\mu(t)>0$, удовлетворяющая (4.1). Будем говорить, что $(R, \lambda)$ включает $(R, \mu)$, если из сходимости $f(t) \rightarrow \bar{f}(R, \mu)$ следует $f(t) \rightarrow \bar{f}(R, \lambda)$. Следующее утверждение дает достаточное условие включения.

TEOPEMA 7. Пусть

$$
\lambda(\tau) \int_{0}^{\tau} \mu d t / \mu(\tau) \int_{0}^{\tau} \lambda d t \rightarrow 0 \quad n p u \quad \tau \rightarrow \infty
$$

и функиия

$$
\lambda(\tau) \int_{0}^{\tau} \mu d t / \mu(\tau)
$$

монотонна при $\tau \geqslant \tau_{0}$. Тогда $(R, \lambda)$ включает $(R, \mu)$.

Пусть функции $\lambda$ и $\mu$ из одного тела Харди и имеют порядок $+\infty$ относительно $t$ :

$$
\frac{\ln \lambda(t)}{\ln t} \rightarrow+\infty \quad \text { и } \quad \frac{\ln \mu(t)}{\ln t} \rightarrow+\infty
$$

при $t \rightarrow+\infty$. Тогда условие (4.2) можно представить в следуюшем виде:

$$
\frac{\dot{\lambda}}{\lambda} / \frac{\dot{\mu}}{\mu} \rightarrow 0
$$

Если $\lambda$ и $\mu$ принадлежат одному телу Харди, то условие о монотонности функции (4.3) при достаточно больших значениях $\tau$ заведомо вьполнено.

ЗАмЕчАнИЕ. Пусть $(R, \lambda(n))$ и $(R, \mu(n))$ - два обычных (дискретных) метода Рисса, причем $\sum \lambda(n)=\sum \mu(n)=\infty$. По теореме Чезаро, если

$$
\frac{\lambda(n+1)}{\lambda(n)} \leqslant \frac{\mu(n+1)}{\mu(n)},
$$

то $(R, \lambda(n))$ включает $(R, \mu(n))$. Если представить $(4.5)$ в эквивалентной форме

$$
\frac{\lambda(n+1)-\lambda(n)}{\lambda(n)} \leqslant \frac{\mu(n+1)-\mu(n)}{\mu(n)},
$$

то становится ясньм (ср. с (4.4)), что теорема 7 - непрерьвный аналог теоремы Чезаpo.

ПРИМЕР. Положим $R_{\alpha}=\left(R, \exp t^{\alpha}\right), \alpha \geqslant 0$. Ясно, что $R_{0}$ является классическим методом Чезаро. Согласно теореме $7, R_{\alpha}$ включает $R_{\beta}$, если $\beta \geqslant \alpha$. Как будет указано ниже, при $\alpha \geqslant 1$ методы $R_{\alpha}$ эквивалентны обычной сходимости.

Теорема 8. Предположим, что

$$
\lambda(\tau) / \int_{0}^{\tau} \lambda d t \geqslant c=\text { const }>0
$$

$u f, \dot{f}$ ограничены. Тогда из $f(t) \rightarrow \bar{f}(R, \lambda)$ вытекает, что $f(t) \rightarrow \bar{f}$ (в обычном смыblсле).

Для функщий $\lambda$ бесконечного порядка относительно $t$ условие (4.6) эквивалентно следующему:

$$
\dot{\lambda} / \lambda \geqslant c .
$$


В частности, все методы $R_{\alpha}=\left(R, \exp t^{\alpha}\right)$ при $\alpha \geqslant 1$ эквивалентны обычной сходимости.

В известных тауберовых теоремах Винера предполагается, что $f$ ограничена и медленно колеблется. Последнее свойство заведомо вьполнено при условии ограниченности производной. Доказательство теорем 7 и 8 можно найти в [15].

Непрерьвное движение $x: \mathbb{R} \rightarrow \mathbb{T}^{n}$ будем называть $(R, \lambda)$-равномерно распределенным (кратко $(R, \lambda)$-рр), если для любой непрерьвнной функции $f: \mathbb{T}^{n} \rightarrow \mathbb{R}$

$$
\lim _{\tau \rightarrow \infty} \int_{0}^{\tau} \lambda(t) f(x(t)) d t / \int_{0}^{\tau} \lambda(t) d t=\frac{1}{(2 \pi)^{n}} \int_{\mathbb{T}^{n}} f(x) d^{n}(x) .
$$

Это соотношение справедливо и для любой интегрируемой по Риману функции $f$ при условии, что функция $t \mapsto f(x(t))$ интегрируема по Риману на каждом конечном интервале. Если $\lambda(t)=1$, то получим определение равномерного распределения по Вейлю.

Для $(R, \lambda)$-рр достаточно проверить, что

$$
\int_{0}^{\tau} \lambda(t) e^{i(m, x(t))} d t / \int_{0}^{\tau} \lambda(t) d t \rightarrow 0
$$

при $\tau \rightarrow \infty$ для всех $m \in \mathbb{Z}^{n} \backslash\{0\}$ (обобщенньй критерий Вейля).

Теорема 9. Пусть $x_{1}(t), \ldots, x_{n}(t)$ - такие $n$ многочленов, что никакая их целочисленная нетривиальная комбиначия не сводится к константе. Если

$$
\lambda(\tau) / \int_{0}^{\tau} \lambda(t) d t \rightarrow 0
$$

и функции $\lambda$ и $t$ принадлежат одному телу Харди, то движение

$$
x_{j}=x_{j}(t), \quad 1 \leqslant j \leqslant n,
$$

будет $(R, \lambda)-p p$ на $\mathbb{T}^{n}$.

Условия этого утверждения заведомо выполнены, если, например, $\lambda=\exp t^{\alpha}$, $\alpha<1$. Таким образом, теорема 9 из [15] - это усиление результата Вейля (теорема 8 из [5], с. 69) о равномерном распределении движения (4.7) на $\mathbb{T}^{n}$ в обычном смысле (когда $\lambda=1$ ).

Обсудим теперь задачу о распределении логарифмов. Пусть сначала $n=1$. Движение $x=\ln t, t \geqslant a>0$, не будет рр на окружности по Вейлю. Действительно,

$$
\frac{1}{\tau} \int_{a}^{\tau} e^{i \ln t} d t=\frac{e^{i \ln \tau}}{1+i}+\frac{\text { const }}{\tau},
$$

что осциллирует и не стремится к нулю при $\tau \rightarrow \infty$. Наоборот, при всех $\varepsilon>0$ движение $x=\ln ^{1+\varepsilon} t$ будет рр по Вейлю на окружности $\mathbb{T}=\{x \bmod 2 \pi\}$.

С другой стороны, поскольку при всех целых $m \neq 0$

$$
\int_{a}^{\tau} \frac{e^{i m \ln t}}{t} d t=\frac{e^{i \ln \tau}}{i m}+\mathrm{const}=O(1),
$$

то движение $x=\ln t$ будет $(R, 1 / t)$-рр. Конечно, $(R, 1 / t)$-метод сильнее метода Чезаpo.

Эти замечания можно обобщить. Справедлива следующая теорема. 
ТЕОрема 10. Если функиия $t \lambda(t)$ монотонна при $t \geqslant t_{0} u$

$$
\tau \lambda(\tau) / \int_{0}^{\tau} \lambda(t) d t \rightarrow 0
$$

то движение $x_{j}=\omega_{j} \ln t$ с рачионально несоизмеримыми $\omega_{1}, \ldots, \omega_{n}$ будет $(R, \lambda)$-рр на $\mathbb{T}^{n}$.

Аналогичное утверждение справедливо и для повторных логарифмов. Например, функция $\ln \ln t$ будет $\left(R,(t \ln t)^{-1}\right)$-рр на окружности $\{x \bmod 2 \pi\}$.

Пусть $f: \mathbb{T}^{n} \rightarrow \mathbb{R}$ - непрерывная функция с нулевым средним значением, $\omega_{1}, \ldots, \omega_{n}$ - набор постоянных частот (не обязательно нерезонансный). Рассмотрим интеграл

$$
I\left(\tau, x_{0}\right)=\int_{0}^{\tau} f\left(\omega_{1} t+x_{1}^{0}, \ldots, \omega_{n} t+x_{n}^{0}\right) d t .
$$

В [16] доказано, что при этих предположениях всегда найдется набор начальньх фаз $x_{0}=\left(x_{1}^{0}, \ldots, x_{n}^{0}\right), f\left(x_{0}\right)=0$, для которых $I\left(\tau, x_{0}\right) \geqslant 0$ для всех $\tau \in \mathbb{R}$. Аналогичное заключение, конечно, справедливо и для неравенства $I\left(\tau, x_{0}\right) \leqslant 0$. Этот результат допускает обобщение [15].

ТеОРема 11. Пусть $\lambda(t)>0$ - невозрастающая функиия. Тогда при сделанных выше предположениях

$$
\int_{0}^{\tau} \lambda(t) f\left(\omega t+x_{0}\right) d t \geqslant 0 \quad(\leqslant 0)
$$

при всех $\tau$ для некоторых начальных фаз $x_{0}$ таких, что $f\left(x_{0}\right)=0$.

СледСтВИЕ 1. Пусть $f$ - непрерывная функиия на $\mathbb{T}^{n}$ с нулевьм.м средним и $p \geqslant 1$. Тогда найдется $x_{0} \in \mathbb{T}^{n}$ такое, что

$$
\int_{0}^{\tau} f\left(\omega t^{p}+x_{0}\right) d t \geqslant 0 \quad(\leqslant 0)
$$

для всех $\tau$.

Достаточно вьполнить замену переменной по формуле $t^{p}=z$.

СлЕДСТВИЕ 2. Пусть $f$ - непрерывная функиия с нулевым средним и произведение $e^{z} \lambda\left(e^{z}\right)$ не возрастает. Тогда найдется $x_{0} \in \mathbb{T}^{n}$ такое, что

$$
\int_{1}^{\tau} \lambda(t) f\left(\omega \ln t+x_{0}\right) d t \geqslant 0 \quad(\leqslant 0)
$$

при всех $\tau$.

Достаточно перейти к новому переменному $z=\ln t$. В частности, заключение следствия 2 справедливо для $\lambda=1 / t$. Если не накладьвать ограничений на функцию $\lambda$, то свойство знакоопределенности теряется. Например, при всех $x_{0}$ интеграл

$$
\int_{1}^{\tau} \cos \left(\ln t+x_{0}\right) d t
$$

бесконечно много раз меняет знак, когда $\tau \rightarrow \infty$.

Пусть теперь $f: \mathbb{T}^{n} \rightarrow \mathbb{R}$ - непостоянная бесконечно дифферениируемая функция с нулевым средним значением, а частоты $\omega_{1}, \ldots, \omega_{n}$ рационально независимы. Как доказано в [17], если $f\left(x_{0}\right) \neq 0$, то интеграл

$$
\int_{0}^{\tau} f\left(\omega t+x_{0}\right) d t
$$

бесконечно много раз меняет знак, когда $\tau \rightarrow \infty$. При $n=1$ это очевидно, а при $n=2$ - доказано в [16]. 
ТеОРема 12. Пусть $\lambda(t)>0$ - неубьвающая функиия. Если все нули функиии

$$
\tau \mapsto \int_{0}^{\tau} \lambda(t) f\left(\omega t+x_{0}\right) d t
$$

простыле, то их бесконечно много.

Если функция $\lambda$ убьвает, то интеграл (4.8) может иметь лишь конечное число нулей. Простым примером служит классический интеграл $\Phi$ ренеля

$$
\int_{0}^{\tau} \cos t^{2} d t
$$

Заменой $t^{2}=z$ он приводится к виду (4.8), причем $\lambda$ монотонно стремится к нулю. Хорошо известно, что функция (4.9) имеет всего один простой нуль $\tau=0$.

\section{$\S 5$. Строгая эргодичность}

Пусть $M$ - компактное метрическое пространство, $T$ - гомеоморфизм $M$. По теореме Крылова-Боголюбова, $T$ сохраняет некоторую борелевскую меру $\mu$ на $M$. Если нормированная $(\mu(M)=1)$ инвариантная относительно $T$ борелевская мера единственна, то непрерьвное преобразование $T$ назьвается строго эргодическим.

Ясно, что строго эргодический гомеоморфизм $T$ эргодичен по отношению к своей единственной инвариантной борелевской мере $\mu$. Однако далеко не каждое непрерьвное эргодическое преобразование будет строго эргодическим. Например, у строго эргодического преобразования нет периодических точек. В противном случае имеется дополнительная инвариантная борелевская мера, сосредоточенная на периодической траектории гомеоморфизма $T$.

Укажем характеристическое свойство строго эргодического преобразования ( $m e$ орема Окстоби [18]): для любой непрерьвной функции $f: M \rightarrow \mathbb{R}$

$$
f\left(T^{n} x\right) \rightrightarrows \int_{M} f d \mu \quad(C)
$$

Символ $\rightrightarrows$ обозначает равномерную сходимость. Ввиду обратимости $T$, соотношение (5.1) справедливо и при $n \rightarrow-\infty$.

ПримеР. Пусть $T$ - гомеоморфизм окружности $M=\{x \bmod 2 \pi\}$, сохраняющий ориентацию. Ясно, что $T x=x+f(x)$, где $f$ - непрерывная $2 \pi$-периодическая функция. Как показал Пуанкаре, для всех $x$

$$
f\left(T^{n} x\right) \rightarrow 2 \pi \lambda \quad(C) .
$$

Число $\lambda$ назьвается иислом вращения гомеоморфизма $T$. Если $T$ не имеет периодических точек, то оно строго эргодическое (см., например, [19]).

Соотношение (5.1) можно распространить на более широкий класс функций. Будем говорить, что функция $f: M \rightarrow \mathbb{R}$ является $\mathscr{R}$-интегрируемой (относительно нормированной борелевской меры $\mu$ на $M$ ), если для любого $\varepsilon>0$ найдутся две непрерьвные функции $f_{1}$ и $f_{2}$ такие, что

$$
\begin{gathered}
f_{1}(x) \leqslant f(x) \leqslant f_{2}(x) \text { для всех } x \in M, \\
\int_{M}\left(f_{2}-f_{1}\right) d \mu<\varepsilon .
\end{gathered}
$$


Очевидно, что $\mathscr{R}$-интегрируемые функции ограничены и все непрерьвные функции $\mathscr{R}$-интегрируемы.

Интеграл $\mathscr{R}$-интегрируемой функции определяется следующим естественным образом. Рассмотрим последовательность $\varepsilon_{n}$, стремяшуюся к нулю. Соответствуюшие последовательности непрерывных функций, удовлетворяющих условиям (5.3), обозначим $f_{1}^{(n)}$ и $f_{2}^{(n)}$. Легко показать, что пределы последовательностей интегралов от $f_{1}^{(n)}$ и $f_{2}^{(n)}$ по мере $\mu$ при $n \rightarrow \infty$ сушествуют и совпадают; это число будем назьвать $\mathscr{R}$-интегралом $f$ по борелевской мере $\mu$ и обозначать

$$
\int_{M} f d \mu
$$

Нетрудно убедиться в корректности определения $\mathscr{R}$-интеграла: оно не зависит от выбора последовательностей $\varepsilon_{n}, f_{1}^{(n)}, f_{2}^{(n)}$.

Пусть, например, $M$ является многомерньм тором $\mathbb{T}^{k}$, а $\mu$-стандартная мера на $\mathbb{T}^{k}$. В этом случае класс $\mathscr{R}$-интегрируемых функций будет совпадать с классом функций, интегрируемых по Риману.

Теорему Окстоби можно слегка обобшить [20].

Теорема 13. Преобразование T строго әргодическое тогда и только тогда, когда для любой $\mathscr{R}$-интегрируемой функции $f$

$$
f\left(T^{n} x\right) \rightrightarrows \int_{M} f d \mu \quad(C)
$$

Подмножество $D \subset M$ будем называть $\mathscr{R}$-измеримым, если его характеристическая функция $f=1_{D}$ будет $\mathscr{R}$-интегрируемой. Положим

$$
\operatorname{mes} D=\int_{M} 1_{D} d \mu \text {. }
$$

Ясно, что mes $M=1$ ввиду предположения о нормированности меры $\mu$.

В качестве функции $f$ из теоремы 1 можно взять характеристическую функцию любой $\mathscr{R}$-измеримой области $D$. Введем последовательность $s_{n}(n \geqslant 0)$ по следующему правилу: $s_{k}=1$, если $x_{k}=T^{k} x \in D$, и $s_{k}=0$ в противном случае. Пусть $\nu(n)=\sum_{0}^{n-1} s_{k}$. Тогда для строго эргодического преобразования будем иметь

$$
\lim _{n \rightarrow \infty} \frac{\nu(n)}{n}=\operatorname{mes} D .
$$

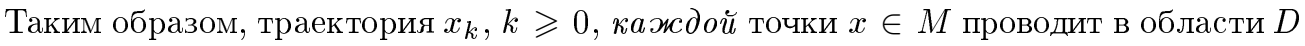
время, пропорциональное в среднем мере этой области. Это - общее определение равномерно распределенной последовательности по Вейлю (см., например, [21]).

Если только что введенная последовательность $s_{k}$ сходится к mes $D$ с точки зрения линейного регулярного метода $S$ при любом выборе $\mathscr{R}$-измеримой области $D$, то последовательность точек $x_{k} \in M, k \geqslant 0$, естественно назвать $S$-равномерно распределенной (кратко $S$-рр).

Следуя [20], покажем, как можно усилить теорему Окстоби с использованием методов суммирования Рисса и Вороного, включенньх в метод Чезаро. 
ТЕОРема 14. Пусть $T$ - строго эргодический гомеоморфизм компактного метрического пространства $M$ и $f$ - R-интегрируемая функиия на $M$. Если

mo

$$
\frac{p_{0}+\left|p_{1}-p_{0}\right|+\cdots+\left|p_{n}-p_{n-1}\right|+p_{n}}{p_{0}+p_{1}+\cdots+p_{n}} \rightarrow 0,
$$

$$
f\left(T^{n} x\right) \rightrightarrows \int_{M} f d \mu \quad\left(R, p_{n}\right) \quad u \quad\left(W, p_{n}\right)
$$

Условие (5.4) заведомо выполнено, если

a) $p_{n+1} \leqslant p_{n} u \sum p_{n}=\infty$,

b) $p_{n+1} \geqslant p_{n} u$

$$
\frac{p_{n}}{p_{0}+\cdots+p_{n}} \rightarrow 0
$$

ПримеР. Пусть $T$ - сохраняющий ориентацию гомеоморфизм окружности без периодических точек. Тогда предельное соотношение (5.2) можно усилить, заменяя метод Чезаро сколь угодно слабыми методами Рисса и Вороного. В [22] этот факт отмечен в общем случае, когда у гомеоморфизма $T$ могут быть периодические точки.

В качестве еще одного примера рассмотрим сложный косой сдвиг на $k$-мерном торе $\mathbb{T}^{k}=\left\{x_{1}, \ldots, x_{k} \bmod 1\right\}$, задаваемьй формулой

$$
\begin{gathered}
T x=\left(\left(x_{1}+\alpha\right) \bmod 1,\left(x_{2}+p_{2,1} x_{1}\right) \bmod 1, \ldots,\right. \\
\left.\left(x_{k}+p_{k, 1} x_{1}+\cdots+p_{k, k-1} x_{k-1}\right) \bmod 1\right),
\end{gathered}
$$

где $\alpha, p_{i, j}$ - вешественные числа. Это преобразование, очевидно, сохраняет стандартную меру на $\mathbb{T}^{k}$. Как показано в [19], если $\alpha$ иррационально, а $p_{j, j-1} \neq 0$ для всех $2 \leqslant j \leqslant k$, то преобразование (5.7) строго эргодично. Следовательно, для каждой точки $x \in \mathbb{T}^{k}$ ее траектория (последовательность точек $T^{n} x, n \geqslant 0$ ) равномерно распределена по Вейлю.

Пусть $\pi_{j}: \mathbb{T}^{k} \rightarrow\left\{x_{j} \bmod 1\right\}$ - естественная проекция $k$-мерного тора на окружность

$$
\pi_{j}\left(x_{1}, \ldots, x_{k}\right)=x_{j} .
$$

Ясно, что каждая траектория $T^{n} x, n \geqslant 0$, при проектировании $\pi_{j}$ переходит в последовательность точек, равномерно распределенную по модулю 1. Чтобы убедиться в этом, достаточно в качестве $f$ взять функции, периодически зависящие лишш от одной переменной $x_{j}$.

Положим теперь $x=0$. Можно доказать (см. [19], [23]), что

$$
\left.\pi_{k}\left(T^{n} x\right)\right|_{x=0}=\{P(n)\},
$$

где $P(z)$ - многочлен от $z$ степени $k$, причем коэффициент при $z^{k}$ иррационален, если $\alpha$ иррационально. Верно и обратное утверждение: для любого многочлена $P$ степени $k$ с иррашиональньм коэффициентом при старшей степени найдется сложный косой сдвиг $k$-мерного тора вида (5.7), у которого число $\alpha$ иррационально, $p_{j, j-1} \neq 0$ и справедлива формула (5.8). Отсюда (по Г. Фюрстенбергу [19]) вытекает замечательньй результат Вейля о равномерном распределении дробных частей многочлена со старшим иррациональным коэффищиентом. Общий случай, когда имеется иррациональньй коэффициент при $z^{r}, r \geqslant 1$, легко сводится к этому случаю. Первоначальное доказательство Вейля основано на совершенно других идеях (см. [5]).

Используя теорему 14 , редукцию Фюстенберга и условие строгой эргодичности косого сдвига (5.7), приходим к следующему усилению теоремы Вейля [20]. 
ТеОРема 15. Пусть $P(z)=a_{0} z^{k}+a_{1} z^{k-1}+\cdots+a_{k}-$ многочлен, у которого один из коэффициентов $a_{0}, a_{1}, \ldots, a_{k-1}-$ иррациональное число. Если $q_{n+1} \leqslant q_{n}$ $u \sum q_{n}=\infty$, то последовательность $\{P(n)\}$ будет $\left(W, q_{n}\right)-p p ;$ если $p_{n+1} \geqslant p_{n} u$ выполнено (5.6), то последовательность $\{P(n)\}$ будет $\left(R, p_{n}\right)$.

Для многочлена первой степени теорема 15 доказана в работах [24], [25].

В заключение этого параграфа дополним теорему Окстоби еше одним утверждением, касаюшимся поведения суммы

$$
\sigma_{n}(x)=f(x)+f(T x)+\cdots+f\left(T^{n-1} x\right), \quad x \in M .
$$

ТЕОРема 16. Пусть T - строго әргодический гомеоморфизм компактного метрического пространства $M u f$ - непрерьвная функиия на $M$. Тогда найдется точка $x_{+}\left(x_{-}\right)$на $M$ такая, что

$$
\sigma_{n}\left(x_{+}\right)-n \int_{M} f d \mu \geqslant 0 \quad\left(\sigma_{n}\left(x_{-}\right)-n \int_{M} f d \mu \leqslant 0\right)
$$

для всех чельх $n$.

Это утверждение, отмеченное в [20], легко распространяется на средние Рисса (Вороного) с невозрастаюшими (неубьваюшими) весами. В таком виде оно является обобщением теоремы 11.

С другой стороны, если непрерьвная функция $f$ не постоянна, то для почти всех $x \in M$ разность

$$
\sigma_{n}(x)-n \int_{M} f d \mu
$$

бесконечно много раз меняет знак при $n \rightarrow \infty$. Это - следствие одного общего результата, установленного в работе [26] для общих эргодических преобразований. Интересно отметить, что множество точек $x \in M$, для которых разность (5.10) меняет знак лишь конечное число раз, может оказаться всюду плотным в $M$. Соответствуюший пример для эргодического поворота окружности указан в [16]. В этом примере $f$ - непрерьвная, но нигде не дифференцируемая функция. Теорема об осцилляциях разности (5.10) обобшена в работе [27] для средних Рисса (Вороного) с неубьваюшими (невозрастающими) весами.

\section{§ 6. Весовые средние и усиленный закон больших чисел}

Для выражения закона больших чисел в теории вероятностей обычно используют сходимость по Чезаро. Однако применение весовых средних придает этому кругу вопросов бо́льшую гибкость. С одной стороны, использование методов суммирования Рисса и Вороного, включенных в метод Чезаро, позволяет усилить классические результаты, с другой - применение методов, которые включают метод Чезаро, позволяет расширить сферу применимости закона больших чисел.

Рассмотрим последовательность случайных величин $\xi_{1}, \xi_{2}, \ldots$ с нулевыми математическими ожиданиями и конечными дисперсиями $\sigma_{1}^{2}, \sigma_{2}^{2}, \ldots$ Эти величины не предполагаются независимыми, и поэтому их попарные ковариации $R_{i, j}=\operatorname{cov}\left(\xi_{i}, \xi_{j}\right)=$ $\mathrm{E}\left(\xi_{i} \xi_{j}\right)$, вообще говоря, отличны от нуля. Классическая теорема Бернштейна [28] утверждает, что если

1) дисперсии $\sigma_{n}^{2}$ ограничены,

2) $\left|R_{i, j}\right| \leqslant \varphi(|i-j|)$, причем $\varphi(m) \rightarrow 0$ при $m \rightarrow \infty$, 
то для любого $\varepsilon>0$

$$
\mathrm{P}\left\{\left|\frac{\xi_{1}+\cdots+\xi_{n}}{n}\right| \geqslant \varepsilon\right\} \rightarrow 0
$$

при $n \rightarrow \infty$.

Условия 1) и 2) можно несколько ослабить, заменив их следуюшими:

$$
\begin{gathered}
\sigma_{1}^{2}+\cdots+\sigma_{n}^{2}=o\left(n^{2}\right) \\
\varphi(n) \rightarrow 0 \quad(C) .
\end{gathered}
$$

Итак, из (6.2) и (6.3) следует (6.1). Именно это утверждение мы и собираемся обобшить [29].

TeOpema 17. Ecлu

$$
\frac{p_{1}^{2} \sigma_{1}^{2}+\cdots+p_{n}^{2} \sigma_{n}^{2}}{\left(p_{1}+\cdots+p_{n}\right)^{2}} \rightarrow 0
$$

$u$

$$
\frac{p_{1} R_{1, n+1}+\cdots+p_{n} R_{n, n+1}}{p_{1}+\cdots+p_{n}} \rightarrow 0
$$

то для любого $\varepsilon>0$

$$
\mathrm{P}\left\{\left|\frac{p_{1} \xi_{1}+\cdots+p_{n} \xi_{n}}{p_{1}+\cdots+p_{n}}\right| \geqslant \varepsilon\right\} \rightarrow 0
$$

npu $n \rightarrow \infty$.

Условие (6.4) можно заменить более простым:

$$
\frac{\sigma_{n}^{2} p_{n}}{p_{1}+\cdots+p_{n}} \rightarrow 0
$$

Более точно, с помощью теоремы Штольца и предположения

$$
p_{n} / \sum_{1}^{n} p_{j} \rightarrow 0
$$

легко доказать, что из (6.7) следует (6.4).

С другой стороны, предполагая, что $\left|R_{i, j}\right| \leqslant \varphi(|i-j|)$, условие (6.5) можно представить в более простом и универсальном виде:

$$
\varphi(n) \rightarrow 0 \quad\left(W, p_{n}\right)
$$

Условие $(6.8)$ - критерий регулярности $\left(W, p_{n}\right)$-метода. В частности, если $\varphi(n) \rightarrow 0$, то заведомо вьполнено (6.9) и, следовательно, (6.5).

Что нового дает теорема 17? Классическое условие (6.2) означает, что дисперсии $\sigma_{n}^{2}$ могут расти медленнее $n$; точнее, если $\sigma_{n}^{2}=o(n)$, то имеет место $(6.2)$. Если же $\sigma_{n}^{2}=c n(c=$ const $>0)$, то $(6.2)$ не выполняется и хорошо известно, что в этом случае классический закон больших чисел (6.1), вообше говоря, не справедлив даже для независимых случайных величин (см. по этому поводу [30; гл. Х] ).

Положим $p_{n}=1 / n$. Тогда соотношение (6.7) принимает вид $\sigma_{n}^{2}=o(n \ln n)$. В частности, дисперсии могут расти линейно вместе с $n$ и мы имеем дело с законом больших 
чисел в форме (6.6), если, конечно, ковариации $R_{i, j}$ равномерно по $|i-j|$ стремятся к нулю (или, более общо, справедливо условие (6.9) с весовыми коэффициентами $\left.p_{n}=1 / n\right)$.

K обсуждаемому кругу вопросов можно подойти с иной стороны. Рассмотрим случай, когда $\sigma_{1}^{2}=\sigma_{2}^{2}=\cdots$ (например, случайные величины $\xi_{1}, \xi_{2}, \ldots$ одинаково распределены, но зависимы). Тогда условие (6.7) заведомо выполнено ввиду предложения (6.8). Можно рассмотреть случай, когда $p_{n}=\exp n^{\alpha}$, где $0 \leqslant \alpha<1$. С ростом $\alpha$ "сила" соответствуюших $\left(W, p_{n}\right)$-методов возрастает, и поэтому мы будем иметь все более слабые условия на убывание ковариаций $\varphi(n)$. С другой стороны, сила $\left(R, \exp n^{\alpha}\right)$-методов убьвает, и при $\alpha \rightarrow 1$ эти методы неограниченно приближаются к обычной сходимости. Но тогда сходимость по вероятности средних Рисса для случайных величин $\xi_{1}, \xi_{2}, \ldots$ к нулю будет более тонким (а следовательно, и более глубоким) фактом.

Идея обобшения закона больших чисел с использованием методов суммирования, отличным от метода Чезаро, конечно, не нова. Упомянем работы [31], [32], в которых соотношения вида (6.6) доказываются для матричных методов суммирования более общего вида, но при этом случайные величины $\xi_{1}, \xi_{2}, \ldots$ предполагаются независимыми.

Следуя [33], обсудим теперь возможности обобшения усиленного закона больших чисел.

ТЕОРема 18. Пусть $\xi_{1}, \xi_{2}, \ldots$ - одинаково распределенные независимые случайные величины с нулевыми средними, имеющие конечные моменты порядков $\leqslant 2 k$, а числа $p_{1}, p_{2}, \ldots$ таковьи, что

$$
\sum_{n=1}^{\infty}\left[\frac{p_{1}^{2}+\cdots+p_{n}^{2}}{\left(p_{1}+\cdots+p_{n}\right)^{2}}\right]^{k}<\infty .
$$

Тогда

$$
\mathrm{P}\left\{\xi_{n} \rightarrow 0\left(R, p_{n}\right)\right\}=1
$$

СлЕДСТВИЕ. Пусть $\xi_{n}$ имеют конечные моменты всех порядков. Тогда

$$
\xi_{n} \rightarrow 0 \quad\left(R, \exp n^{\alpha}\right) \quad \text { n.н. }
$$

для всех $0 \leqslant \alpha<1$.

ТЕОрема 19. Пусть $\xi_{1}, \xi_{2}, \ldots$ - независимые случайные величины с нулевыми средними значениями и дисперсиями $\sigma_{1}^{2}, \sigma_{2}^{2}, \ldots$ Если $p_{j}>0, \sum p_{j}=\infty u$

$$
\sum \frac{p_{j}^{2} \sigma_{j}^{2}}{\left(p_{1}+\cdots+p_{j}\right)^{2}}<\infty,
$$

mo

$$
\xi_{n} \rightarrow 0 \quad\left(R, p_{n}\right) \quad \text { n.н. }
$$

Ясно, что при $p_{1}=p_{2}=\cdots$ условие (6.10) переходит в классическое условие Колмогорова $\sum \sigma_{n}^{2} / n^{2}<\infty$.

Применим теорему 19 к случаю одинаково распределенных случайных величин. Тогда условие (6.10) примет вид

$$
\sum \frac{p_{n}^{2}}{\left(p_{1}+\cdots+p_{n}\right)^{2}}<\infty
$$


Пусть, например, $p_{n}=\exp n^{\alpha}, \alpha \geqslant 0$. Для этого случая ряд (6.12) сходится лишь при $\alpha<1 / 2$, что является более слабым результатом по сравнению с теоремой 18 . Правда, следует иметь в виду, что в теореме 19 на случайные величины накладьваются более слабые ограничения. Заметим еше, что для $\alpha=1 / 2$ можно привести пример последовательности неограниченных и одинаково распределенных независимых случайных величин $\xi_{1}, \xi_{2}, \ldots$, для которой соотношение (6.11) не вьполняется.

ТЕОРема 20. Пусть $\xi_{1}, \xi_{2}, \ldots$ - независимые случайнье величины с нулевыми математическими ожиданиями и одинаковыми дисперсиями. $Е с л и\left|\xi_{n}\right| \leqslant M$ n.H., $M=$ const, а числа $p_{n}$ таковьи, что

$$
\frac{p_{n}^{2}}{\sum_{1}^{n} p_{k}^{2}}=\frac{\psi(n)}{\ln \ln \sum_{1}^{n} p_{k}^{2}},
$$

$\psi(n) \rightarrow 0$ npu $n \rightarrow \infty$, mo

$$
\xi_{n} \rightarrow 0 \quad\left(R, p_{n}\right) \quad \text { n.н. }
$$

Это утверждение вьводится с помощью закона повторного логарифма ХинчинаКолмогорова.

В частности, если $p_{n}=\exp n^{\alpha}, \alpha<1$, то функция $\psi$ убьвает как

$$
\frac{\ln n}{n^{1-\alpha}} \text {. }
$$

Положим теперь

$$
p_{n}=\exp \frac{n}{\ln ^{\gamma} n}, \quad \gamma>0 .
$$

С помошью формулы суммирования Эйлера-Маклорена нетрудно проверить, что

$$
\frac{\sum p_{k}^{2}}{\left(\sum p_{k}\right)^{2}}
$$

асимптотически убывают с ростом $n$ как $1 / \ln ^{\gamma} n$. Следовательно, для всех $k$ ряды в теореме 18 расходятся. Однако в этом случае

$$
\psi(n) \sim \ln ^{1-\gamma} n .
$$

Таким образом, если $\gamma>1$, то (по теореме 20) для коэффициентов вида (6.13) справедлив усиленный закон больших чисел.

Стоит, наверное, подчеркнуть, что в теореме 20 по сравнению с теоремой 18 накладьваются более сильные условия на случайные величины $\xi_{n}$.

\section{§7. Индивидуальная эргодическая теорема}

Пусть $M-$ пространство с конечной мерой $\mu(\mu(M)<\infty)$ и $T$ - сохраняющее меру (не обязательно обратимое) преобразование: $\mu\left(T^{-1} A\right)=\mu(A)$ для любой измеримой области $A$. По теореме Биркгофа-Хинчина, для $f \in L_{1}$ при почти всех $x \in M$ последовательность $f\left(T^{n} x\right)$ сходится по Чезаро к инвариантной (относительно преобразования $T)$ и интегрируемой функции $\bar{f}(x)$, причем

$$
\int_{M} f d \mu=\int_{M} \bar{f} d \mu .
$$


Этому замечательному результату предшествовала статистическая эргодическая теорема фон Неймана, утверждающая сходимость в среднем

$$
f\left(T^{n} x\right) \rightarrow \bar{f}(x) \quad(C) .
$$

Пусть $L_{2}$ - линейное пространство комплекснозначных функций $f$ на $M$, суммируемых с квадратом. Каждой функции $f$ можно сопоставить функцию $g(x)=f(T x)$, $x \in M$. Хорошо известно, что соответствие $f \mapsto g=U f$ определяет изометрический оператор $U$ в $L_{2}$. Теорема фон Неймана утверждает, что

$$
U^{n} f \rightarrow P f \quad(C),
$$

где $P$ - оператор, проектируюший $L_{2}$ на подпространство всех векторов, инвариантных относительно $U$.

ТеОрема 21. Если $U$ - изометрический оператор в комплексном гильбертовом пространстве и весовые коэффичиенты $p_{n}$ удовлетворяют условию (5.4), mo

$$
U^{n} f \rightarrow P f \quad\left(R, p_{n}\right) \quad u \quad\left(W, p_{n}\right) .
$$

Когда $p_{n}=1$, получаем классическую теорему фон Неймана. Теорема 21 утверждает, что при $n \rightarrow \infty$ функции $f\left(T^{n} x\right)$ сходятся в среднем к инвариантной функции преобразования $T$ в более сильном смысле, чем в теореме фон Неймана.

Теорема 21 остается содержательной, если гильбертово пространство заменить комплексной плоскостью. В этом случае изометрический оператор представляется комплексным числом $z$, по модулю равным 1 . Если $z=1$, то средние Вороного, очевидно, равны единице. Если $z \neq 1$, то теорема 21 утверждает, что

$$
z^{n} \rightarrow 0 \quad\left(W, q_{n}\right) .
$$

В качестве примера рассмотрим случай, когда $q_{n}=1 / n$. Ясно, что

$$
\frac{z^{n}+z^{n-1} / 2+\cdots+1 / n}{1+1 / 2+\cdots+1 / n}=\frac{z^{n+1}\left(z^{-1}+z^{-2} / 2+\cdots+z^{-n-1} / n\right)}{\ln n+O(1)} .
$$

Так как $z^{-1} \neq 1$, то при $n \rightarrow \infty$ выражение в круглых скобках стремится к $\ln \left(1-z^{-1}\right)$. Это однозначная непрерьвная функция на окружности с выколотой точкой $\{z:|z|=1$ и $z \neq 1\}$. Следовательно, (7.1) стремится к нулю как $1 / \ln n$.

В работе [34] рассматривались более обшие методы суммирования, задаваемые бесконечной матрицей из неотрицательных чисел $a_{n, k}(n, k=0,1,2, \ldots)$, причем

$$
\sum_{k=0}^{\infty} a_{n, k}=1
$$

для всех $n$. В [34] указан критерий, когда средние

$$
\sum_{k=0}^{\infty} a_{n, k} U^{k} f
$$

сходятся в среднем к $P f$ :

(1) $\lim _{n \rightarrow \infty} \sum_{k=0}^{\infty} a_{n, k \alpha+j}=1 / \alpha$ для всех $0 \leqslant j \leqslant \alpha$ и $\alpha=2,3,4, \ldots$,

(2) для любого ирращионального $\gamma$ последовательность $\{n \gamma\}$ равномерно распределена относительно этого метода суммирования. 
Заметим, что условие (2) неконструктивно: дело в том, что описание матричных методов, для которых последовательности $\{n \gamma\}, \gamma \notin \mathbb{Q}$, равномерно распределены на отрезке $[0,1]$, является пока нерешенной задачей (см. по этому поводу [21]).

В [34] указаны также достаточные условия на коэффициенты $a_{n, k}$, при которых статистическая эргодическая теорема имеет место для преобразований со слабым и сильным перемешиванием. Результаты другого характера, касаюшиеся обобщения теоремы фон Неймана для весовых средних, можно найти в [35].

Существенно более трудным представляется следуюший вопрос: можно ли усилить индивидуальную эргодическую теорему Биркгофа-Хинчина, заменив суммирование по Чезаро на более слабьй метод суммирования Рисса или Вороного? Как мы видели в $\S 5$, для строго эргодических преобразований ответ положительньй (теорема 14). Однако не исключено, что для точечной сходимости в типичной ситуации ответ может оказаться отрицательным.

Похожая задача рассматривалась в работах Г. Бакстера [36], [37] (см. также [38]) для метода Рисса $\left(R, p_{n}\right)$, когда весовые коэффициенты определяются из рекуррентного соотношения

$$
p_{n}=f_{1} p_{n-1}+\cdots+f_{n} p_{0}, \quad p_{0}=1,
$$

где $f_{k}(k \geqslant 1)$ - заданная последовательность неотрицательных чисел, для которых $\sum f_{k}=1$. Ясно, что все $p_{n}$ принадлежат отрезку $[0,1]$ и $p_{n}$ стремится к конечному пределу, когда $n \rightarrow \infty$. При некоторых дополнительных условиях в [21], [22] доказана индивидуальная эргодическая теорема для $\left(R, p_{n}\right)$-метода. В частности, если $f_{1}=1$, а остальные $f_{k}=0$, то получаем обычную теорему Биркгофа-Хинчина.

Если $p_{n}$ стремятся к конечному пределу, то $\left(R, p_{n}\right)$-метод может оказаться эквивалентным обычному методу Чезаро (такую возможность не исключает сам Бакстер). Поэтому наибольший интерес (согласно [22]) представляет случай, когда $p_{n} \rightarrow 0$. Однако если при этом $p_{n}$ убьвают монотонно, то $\left(R, p_{n}\right)$-метод включает метод Чезаро, и поэтому обобшенная индивидуальная эргодическая теорема сразу вытекает из обычной теоремы Биркгофа-Хинчина. Укажем еше работу [39], в которой рассматривалась сходимость средних Рисса последовательности случайных величин с невозрастаюшими весами $p_{n}$.

Чтобы лучше понять возникаюшие здесь трудности, рассмотрим более детально случай, когда $M$ - единичньй отрезок [0,1], а $T$ - преобразование Бернулли

$$
x \mapsto 2 x \bmod 1 .
$$

Это преобразование необратимое, но сохраняет обычную меру на $[0,1]: \operatorname{mes} L=$ $\operatorname{mes}\left(T^{-1} L\right)$, где $L$ - любое измеримое множество на $[0,1]$, a $T^{-1} L$ - полный прообраз $L$. Поскольку преобразование Бернулли эргодическое, то для любой интегрируемой функции $f$ и для почти всех $x$

$$
f\left(T^{n} x\right) \rightarrow \int_{0}^{1} f(x) d x \quad(C) .
$$

Зададим кусочно-постоянную функцию $f$ следуюшим образом: $f(x)=1$ при $0 \leqslant$ $x<1 / 2$ и $f(x)=-1$ при $1 / 2 \leqslant x \leqslant 1$. Функции $r_{n+1}(x)=f\left(T^{n} x\right), n \geqslant 0$, называются функииями Радемахера. Они образуют ортонормированную систему. В частности, их средние равны нулю, а дисперсии равны единице. Соотношение (7.2) переходит в усиленньй закон больших чисел (по Борелю, см. [40]):

$$
r_{n}(x) \rightarrow 0 \quad(C)
$$

для почти всех $x$. 
Следовательно, предельное соотношение (7.3) можно заменить более сильным

$$
r_{n}(x) \rightarrow 0 \quad\left(R, p_{n}\right)
$$

если весовые коэффищиенты $p_{n}$ удовлетворяют условию теоремы 20 . В частности, (7.4) справедливо для $p_{n}=\exp n^{\alpha}, \alpha<1$. Однако вопрос о справедливости (7.4) для $p_{n}=\exp (n / \ln n)$ сводится к более тонкому анализу закона повторного логарифма.

\section{§ 8. Некоторые нерешенные задачи}

$1^{\circ}$. Какие регулярные матричные методы суммирования $S$ допускают хотя бы одну $S$-рр последовательность? Например, согласно [41] критерий существования $\left(R, p_{n}\right)$-рр последовательности сводится к условию (6.8). Более простая задача: при каких условиях на весовые коэффициенты $p_{n}$ имеется $\left(W, p_{n}\right)$-рр последовательность?

Эти задачи мотивируются следуюшим обобщением теоремы фон Неймана (1925 г.), установленным в [41]: если имеется хотя бы одна $W$-рр последовательность, то любую всюду плотную на $[0,1]$ последовательность можно сделать $W$-рр после подходящего переупорядочивания. Этот результат, по-видимому, справедлив для любых линейных регулярных методов суммирования.

$2^{\circ}$. До сих пор считается нерешенной следующая задача: описать все $S$-методы, для которых последовательности $\{n \alpha\}, \alpha$ - иррационально, будут $S$-рр. Более просто (но также содержательно) эта задача вьглядит для регулярных $R$ - и $W$-методов суммирования. Достаточнылм условием является соотношение (5.4).

$3^{\circ}$. Пусть

$$
P(z)=\alpha z^{k}+a_{k-1} z^{k-1}+\cdots+a_{1} z+a_{0}
$$

- многочлен степени $k \geqslant 1, \alpha$ иррационально, а $x \mapsto f(x)$ - непрерывная периодическая функция с единичным периодом и нулевым средним. Положим

$$
\sigma_{n}(a)=\sum_{j=1}^{n} f(\{P(j)\}), \quad a=\left(a_{0}, \ldots, a_{k-1}\right) .
$$

Имеется много работ, в которых получены нетривиальные оценки таких сумм, когда $f(x)=\sin 2 \pi x$ и $f(x)=\cos 2 \pi x($ см. [42]).

Из результатов, изложенных в $\S 5$, можно вывести следуюшие свойства суммы (8.1):

$1)$ найдутся такие $a_{+}$и $a_{-}$, что $\sigma_{n}\left(a_{+}\right) \geqslant 0$ и $\sigma_{n}\left(a_{-}\right) \leqslant 0$ при всех целых $n$,

2 ) для почти всех $a \in \mathbb{R}^{k}$ сумма $\sigma_{n}(a)$ бесконечно много раз меняет знак, когда $n \rightarrow \infty$.

Последнее означает, что $\sigma_{n}$ не может принимать только положительные или отрицательные значения при $n \geqslant n_{0}$.

Верно ли, что сумма (8.1) обладает свойством возвращаемости: для любого $\varepsilon>0$ и любого $N$ найдется $n>N$ такое, что $\left|\sigma_{n}(a)\right|<\varepsilon ?$ Для $k=1$ это доказано в рабо$\operatorname{tax}[43],[44]$ в предположении, что функция $f$ абсолютно непрерывна. Более того, свойство возвращаемости в этом случае равномерно по параметру $а$. Для непрерьвных функций контрпример указан в [16]. Поэтому при $k>1$ следует ограничиться гладкими функциями.

$4^{\circ}$. Аналогичный вопрос целесообразно рассмотреть для сумм вида

$$
\sum_{j=1}^{n} g(j) F(\{f(j)\})
$$


или

$$
\sum_{j=1}^{n} g(n-j) F(\{f(j)\}),
$$

где $F$ - гладкая периодическая функция с единичным периодом и нулевым средним значением, а $f$ и $g-$ функции из теоремы 3 и теорем $4-5$ соответственно.

$5^{\circ}$. Верно ли, что если $\lambda(t)$ - неубывающая функция и $f\left(x_{0}\right) \neq 0$, то функция $(4.8)$ имеет бесконечно много нулей?

$6^{\circ}$. По теореме И. М. Виноградова, последовательность $\{\alpha p\}$, где $\alpha$ иррационально, а $p$ принимает значения последовательных простых чисел, равномерно распределена в смысле классического определения Вейля. Можно ли усилить этот результат, заменив метод Чезаро более слабым методом Рисса или Вороного? Аналогичньй вопрос относится к последовательности $\{F(p)\}$, где $F$ - многочлен, у которого хотя бы один коэффищиент, кроме свободного члена, иррационален.

$7^{\circ}$. Наконец, сформулируем еще раз главньй вопрос, ради которого, собственно, и была написана эта статья: можно ли в эргодической теореме Биркгофа-Хинчина заменить сходимость по Чезаро более слабой сходимостью по Риссу или Вороному? Вот одно из возможных уточнений этой задачи: верно ли, что

$$
f\left(T^{n} x\right) \rightarrow \bar{f}(x) \quad\left(R, p_{n}\right),
$$

где $p_{n}=\exp n^{\alpha}, 0<\alpha<1 / 2$ ?

\section{СПИСОК ЛИТЕРАТУРЫ}

[1] R. L. Duncan. Note on the initial digit problem // Fibonacci Quart. 1969. V. 7. P. 474-475.

[2] B. J. Flehinger. On the probability that a random number has initial digit $A / /$ Amer. Math. Monthly. 1966. V. 73. P. 1056-1061.

[3] M. Tsuji. On the uniform distribution of numbers mod 1 // J. Math. Soc. Japan. 1952. V. 4. P. 313-322.

[4] F. Benford. The law of anomalous numbers // Proc. Amer. Philos. Soc. 1938. V. 78. P. $551-572$

[5] Г. Вейль. Избранные труды. М.: Наука, 1984.

[6] S. Newcomb. Note on the frequency of use of the different digits in natural numbers // Amer. J. Math. 1881. V. 4. P. 39-40.

[7] P. Diaconis. The distribution of leading digits and uniform distribution mod $1 / /$ Ann. Probab. 1977. V. 5. № 1. P. 72-81.

[8] Н. Бурбаки. Функции действительного переменного. М.: Наука, 1965.

[9] R. A. Raimi. The first digit problem // Amer. Math. Monthly. 1976. V. 83. № 7. P. 521-538.

[10] Г. Полиа, Г. Сеге. Задачи и теоремы из анализа. Т. I. М.: Наука, 1978.

[11] Г. Харди. Расходящиеся ряды. М.: ИЛ, 1951.

[12] В. В. Козлов. О равномерном распределении // Изв. вузов. Сев.-Кавк. регион. Сер. Естеств. науки. 2001. С. 96-99.

[13] С. Б. Гашков, В.Н. Чубариков. Арифметика. Алгоритмы. Сложность вычислений. М.: Наука, 1996.

[14] В.В.Козлов, Т. Мадсен. Равномерное распределение и сходимость по Вороному // Матем. сб. 2005. Т. 196. № 10. С. 103-110.

[15] В. В. Козлов. О равномерном распределении на торе // Вестн. Моск. ун-та. Сер. 1. Матем. мех. 2004. № 2. С. 22-29.

[16] В.В.Козлов. Об интегралах квазипериодических функций // Вестн. Моск. ун-та. Сер. 1. Матем. мех. 1978. № 1. С. 106-115.

[17] Н. Г. Мощевитин. О возвращаемости интеграла гладкой трехчастотной условно-периодической функции // Матем. заметки. 1995. Т. 58. № 5. С. 723-735.

[18] J. C. Oxtoby. Ergodic sets // Bull. Amer. Math. Soc. 1952. V. 58. № 2. P. 116-136.

[19] H. Furstenberg. Strict ergodicity and transformation of the torus // Amer. J. Math. 1961. V. 83. № 4. P. 573-601. 
[20] В. В. Козлов. Весовые средние, строгая эргодичность и равномерное распределение // Матем. заметки. 2005. Т. 78. №3. С. 358-367.

[21] Л. Кейперс, Г. Нидеррейтер. Равномерное распределение последовательностей. М.: Наука, 1985.

[22] В.В.Козлов, Т. Мадсен. Числа вращения Пуанкаре и средние Рисса и Вороного // Матем. заметки. 2003. Т. 74. № 2. С. 314-315.

[23] И.П. Корнфельд, Я.Г. Синай, С.В.Фомин. Эргодическая теория. М.: Наука, 1980.

[24] J. Cigler. Methods of summability and uniform distribution // Compositio Math. 1964. V. 16. P. $44-51$.

[25] A.F. Dowidar. A note on the generalized uniform distribution $(\bmod 1) / /$ J. Natur. Sci. Math. 1971. V. 11. P. 185-189.

[26] G. Halász. Remarks on the remainder in Birkhoff's ergodic theorem // Acta Math. Acad. Sci. Hungar. 1976. V. 28. № 3-4. P. 389-395.

[27] А. А. Сорокин. Об осцилляциях средних Рисса и Вороного // Вестн. Моск. ун-та. Сер. 1. Матем. мех. 2005. № 2. C. 13-17.

[28] С. Н. Бернштейн. О законе болшших чисел // Сообщ. Харьков. матем. об-ва. Сер. 2. 1918. T. 16. C. $82-87$.

[29] В. В. Козлов, Т. Мадсен, А. А. Сорокин. О весовых средних значениях слабозависимых случайных величин // Вестн. Моск. ун-та. Сер. 1. Матем. мех. 2004. № 5. С. 34-37.

[30] В. Феллер. Введение в теорию вероятностей и ее приложения. Т. 1. М.: Мир, 1984.

[31] W. E. Franck, D. L. Hanson. Some results giving rates of convergence in the law of large numbers for weighted sums of independent random variables // Trans. Amer. Math. Soc. 1966. V. 124. № 2. P. 347-359.

[32] D. L. Hanson, F. T. Wright. Some convergence results for weighted sums of independent random variables // Z. Wahrscheinlichkeitstheor. verw. Geb. 1971. V. 19. P. 81-89.

[33] В.В. Козлов. Суммирование расходящихся рядов и эргодические теоремы // Труды семин. им. И. Г. Петровского. 2002. Вып. 22. С. 142-168.

[34] D. L. Hanson, G. Pledger. On the mean ergodic theorem for weighted averages // Z. Wahrscheinlichkeitstheor. verw. Geb. 1969. V. 13. № 2. P. 141-149.

[35] L. W. Cohen. On the mean ergodic theorem // Ann. of Math. (2). 1940. V. 41. P. 505-509.

[36] G. Baxter. An ergodic theorem with weighted averages // J. Math. Mech. 1964. V. 13. № 3. P. 481-488.

[37] G. Baxter. A general ergodic theorem with weighted averages // J. Math. Mech. 1965. V. 14. № 2. P. 277-288.

[38] R. V. Chacon. Ordinary means imply recurrent means // Bull. Amer. Math. Soc. 1964. V. 70. P. $796-797$.

[39] В. Ф. Гапошкин. О суммировании стационарных последовательностей методами Рисса // Матем. заметки. 1995. Т. 57. № 5. С. 653-662.

[40] М. Кац. Статистическая независимость в теории вероятностей, анализе и теории чисел. М.: ИЛ, 1963.

[41] Е. В. Горделий. К теореме фон Неймана о перестановках всюду плотных последовательностей // Вестн. Моск. ун-та. Сер. 1. Матем. мех. 2004. №6. С. 18-24.

[42] Ло-ген Хуа. Метод тригонометрических сумм и его применения в теории чисел. М.: Мир, 1964.

[43] В. В. Козлов. Об одной задаче Пуанкаре // ПММ. 1976. Т. 40. № 2. С. 352-355.

[44] Е. А. Сидоров. Об условиях равномерной устойчивости по Пуассону цилиндрических систем // УМН. 1979. Т. 34. №6. С. 184-188. 